\title{
Positive Stability Analysis and Bio-Circuit Design for Nonlinear Biochemical Networks
}

\author{
Yonghui Sun, Zhinong Wei, and Guoqiang Sun \\ College of Energy and Electrical Engineering, Hohai University, Nanjing 210098, China \\ Correspondence should be addressed to Yonghui Sun; sunyonghuil68@gmail.com
}

Received 4 February 2013; Accepted 24 March 2013

Academic Editor: Chuangxia Huang

Copyright (C) 2013 Yonghui Sun et al. This is an open access article distributed under the Creative Commons Attribution License, which permits unrestricted use, distribution, and reproduction in any medium, provided the original work is properly cited.

This paper is concerned with positive stability analysis and bio-circuits design for nonlinear biochemical networks. A fuzzy interpolation approach is employed to approximate nonlinear biochemical networks. Based on the Lyapunov stability theory, sufficient conditions are developed to guarantee the equilibrium points of nonlinear biochemical networks to be positive and asymptotically stable. In addition, a constrained bio-circuits design with positive control input is also considered. It is shown that the conditions can be formulated as a solution to a convex optimization problem, which can be easily facilitated by using the Matlab LMI control toolbox. Finally, a real biochemical network model is provided to illustrate the effectiveness and validity of the obtained results.

\section{Introduction}

In the past decades, biochemical networks, such as metabolic networks [1] and genetic networks [2], have received considerable attention and become a hot research topic [3-5]. A great number of results have been obtained, such as gene expression data modeling [6-9] and dynamic analysis of biochemical networks $[10,11]$.

It is not surprising that dynamical system theory plays a central role in understanding biological and physiological processes $[4,12]$ since it provides a powerful tool to quantitatively analyze these biochemical networks from a systematic viewpoint. In addition, dynamical system theory is important and useful for the development of synthetic and systems biology, which has a great potential in gene therapy and drug design [13]

It is worth noting that dynamical models of many biological and physiological processes, such as metabolic systems and endocrine systems [1], biochemical reactions [12], are derived from mass and energy balance considerations that take nonnegative chemical concentrations as dynamic states. Hence, state trajectories of such biochemical systems remain in the nonnegative orthant of the state space for arbitrary nonnegative initial conditions. Such systems are commonly referred to as nonnegative systems or positive systems in the literature [14-17]. In this paper, we call them positive systems for convenience. A subclass of positive dynamical systems are compartmental systems [18], which involve dynamical models that are characterized by conservation laws (e.g., mass and energy) capturing exchange of materials between coupled macroscopic subsystems known as compartments. Each compartment is assumed to be kinetically homogeneous. That is, any material entering the compartment is instantaneously mixed with materials of the compartment. There have been some studies on this kind of systems with application to biochemical networks $[4,19]$.

Recently, there have been some results on stability analysis of gene networks with some special regulation functions [20-25]. However, due to nonlinearity and complexity of biochemical networks, there does not exist a systematic approach to stability analysis of such nonlinear biochemical networks. It is noted that the fuzzy interpolation approach proposed in [26] can be seen as a promising way of dealing with nonlinear complex systems. In [27], the authors firstly used the fuzzy approximation method to investigate the robust stability of stochastic biochemical regulatory networks, where, however, the positive constraint of network states was ignored.

On the other hand, there have been some other results on bio-circuits design for biochemical networks. In [28], an external optimal control input was applied to stabilize a gene regulatory system. In [29], a simple robust circuit has been designed for the S-system model without considering 
stochastic noises. In [30], a robust engineering principle was proposed for stochastic biochemical regulatory networks with parameter uncertainties and disturbances. However, most of the aforementioned results paid little attention to the positive constraint of the states of biochemical networks, not to mention constrained bio-circuits with positive control input. In many cases, the states of those studied systems would become negative in simulation, which could be seen as the main drawbacks of these results.

In this paper, the T-S fuzzy system is employed to approximate nonlinear biochemical networks by interpolating several local linear systems. A positive stability condition and a bio-circuits design procedure will be developed for the nonlinear biochemical networks. In addition, a constrained bio-circuits design with positive control input will also be considered. Finally, a real biochemical network is given to illustrate the effectiveness of the obtained results.

The rest of the paper is organized as follows. In Section 2, some useful definitions and lemmas for positive systems are introduced. In Section 3, by using the fuzzy approximation approach, a sufficient condition for positive stability will be derived for nonlinear biochemical networks. In Section 4, bio-circuits design will be developed from a systematic point of view. In addition, a constrained bio-circuits design with positive control input will also be considered. In Section 5, a real biochemical network is given to illustrate the effectiveness of the obtained results. Finally, the paper will be closed with a conclusion.

\section{Notation and Preliminaries}

Notation. $\mathbb{R}$ denotes the set of real numbers, $\mathbb{R}^{n}$ stands for the vector space of all $n$-tuples of real numbers, and $\mathbb{R}^{n \times m}$ is the space of $n \times m$ matrices with real entries. For $x$ in $\mathbb{R}^{n}, x_{i}$ denotes the $i$ th component of $x . \mathbb{R}_{+}^{n \times m}$ denotes the sets of all $n \times m$ real matrices with nonnegative entries and $\mathbb{R}_{+}^{n} \triangleq\{x \in$ $\left.\mathbb{R}^{n}: x \geq 0\right\}$. For a real matrix $A, A \geq 0(>0)$ means that all its entries are nonnegative (positive). $A^{\mathrm{T}}$ is the transpose of $A$, and $A^{-1}$ is the inverse of $A$. The notation $P>0$ means that $P$ is symmetric and positive definite. The following notations of matrices are used throughout this paper: $A_{i}=\left[a_{k j}^{i}\right], B_{i}=$ $\left[b_{1}^{i} ; b_{2}^{i} ; \ldots ; b_{n}^{i}\right]$.

Consider a general nonlinear system

$$
\begin{gathered}
\frac{d x}{d t}=f(x(t), u(t)), \\
x(0)=x_{0},
\end{gathered}
$$

where $x(t) \in \mathbb{R}^{n}$ is the state vector and $u(t) \in \mathbb{R}^{m}$ is the control input. The nonlinear function $f(x(t), u(t))$ satisfies $f(0,0)=0$ and $f \in \mathscr{C}^{2}$; that is, $f$ has the second-order continuous derivative with respect to $x$ and $u$.

Firstly, some definitions and useful lemmas for positive nonlinear systems are given as follows.

Definition 1. Given any positive initial condition $x(0)=x_{0} \in$ $\mathbb{R}_{+}^{n}$, the unforced nonlinear system (1) is said to be positive if the corresponding trajectory $x(t) \in \mathbb{R}_{+}^{n}$ for all $t \geq 0$.
Definition 2. Let $f=\left[f_{1}, f_{2}, \ldots, f_{n}\right]^{\mathrm{T}}: \mathscr{D} \subseteq \mathbb{R}_{+}^{n} \rightarrow \mathbb{R}^{n}$ the nonlinear function $\mathrm{f}$ is positive (or essentially nonnegative in [19]) if $f_{i}(x(t)) \geq 0$, for all $i=1,2, \ldots, n$ and $x(t) \in \mathscr{D} \subseteq \mathbb{R}_{+}^{n}$.

Lemma 3 (see [19]). Consider the unforced nonlinear system (1). If $f$ is positive and continuously differentiable in $\mathscr{D} \subseteq \mathbb{R}_{+}^{n}$ and $f(0)=0$, then $A \triangleq \partial f /\left.\partial x\right|_{x=0}$ is positive (essentially nonnegative).

Lemma 3 implies that if a nonlinear system is positive, then its linearization is also positive.

Theorem 4. For the unforced system (1), if the nonlinear function $f$ is positive, and if there exists a Lyapunov function $V(x(t))>0$ and $V(0)=0$ satisfying the following inequality:

$$
\left(\frac{\partial V(x(t))}{\partial x}\right)^{\mathrm{T}} f(x(t))<0
$$

for all nonzero $x(t) \in \mathbb{R}_{+}^{n}$, then the equilibrium point $x(t)=0$ of the nonlinear system (1) is asymptotically stable.

As a special case of the nonlinear system (1), the following linear system is considered

$$
\begin{gathered}
\frac{d x}{d t}=A x(t)+B u(t), \\
x(0)=x_{0}
\end{gathered}
$$

where $A \in \mathbb{R}^{n \times n}$ and $B \in \mathbb{R}^{n \times m}$. Some useful results from [14] are presented as follows.

Definition 5. A real matrix $M$ is called a Metzler matrix if its off-diagonal elements are nonnegative, that is

$$
M_{i j} \geq 0, \quad i \neq j .
$$

Lemma 6. The unforced linear system (3) is positive if and only if $A$ is a Metzler matrix.

Lemma 7. The unforced positive linear system (3) is asymptotically stable if and only if there exists a positive definite diagonal matrix $P$ such that

$$
P A+A^{\mathrm{T}} P<0
$$

Remark 8. It follows from the physical consideration that all the states of biochemical networks should stay in the positive orthant. Thus, positive systems are suitable for quantitatively studying biochemical networks, such as gene microarray data modeling [6-8] and bio-circuits design for biochemical networks $[3,4]$.

\section{Positive Stability Analysis of Nonlinear Biochemical Networks}

As pointed out in Introduction, many applications in biochemical processes give rise to nonlinear dynamical systems, such as genetic networks, metabolic pathways and membrane transports, to cite just a few examples. Consider the nonlinear 
system (1) for representation of biochemical networks, which describes complex interactions between molecules. It is noted that the states of the system denote the concentrations of the molecules, the nonlinear function $f$ is the regulation function and the control input $u(t)$ can be seen as the external sources, such as drugs, proteins, or other chemical complexes.

For a general nonlinear biochemical network (1), it is very difficult if not impossible to find a suitable Lyapunov function $V$ such that the condition (2) is satisfied, especially when the positive constraint of the states should be also maintained. However, the T-S fuzzy interpolation approach provides a way to approximate the nonlinear biochemical network and, thus, potentially provides a simplified method for positive stability analysis and bio-circuits design.

Consider a nonlinear biochemical network described by a T-S fuzzy system

$$
\begin{gathered}
R^{i}: \operatorname{IF} z_{1}(t) \text { is } M_{1}^{i} \text { and } \cdots z_{r}(t) \text { is } M_{r}^{i} \text {, THEN } \\
\dot{x}(t)=A_{i} x(t)+B_{i} u(t), \\
y(t)=C_{i} x(t),
\end{gathered}
$$

where $i=1,2, \ldots, L$ and $L$ is the number of fuzzy rules; $z_{1}(t), z_{2}(t), \ldots, z_{r}(t)$ are the premise variables and $M_{l}^{i}(i=$ $1,2, \ldots, L, l=1,2, \ldots, r)$ are the fuzzy sets; $u(t)$ and $y(t)$ are the control input and output, respectively; $A_{i}, B_{i}$, and $C_{i}$ are the known matrices of appropriate dimensions.

By using a center average defuzzifier, product inference, and a singleton fuzzifier, the global dynamics of the T-S fuzzy system (6) cab be described by

$$
\begin{gathered}
\dot{x}(t)=\sum_{i=1}^{L} \alpha_{i}(z(t))\left(A_{i} x(t)+B_{i} u(t)\right), \\
y(t)=\sum_{i=1}^{L} \alpha_{i}(z(t)) C_{i} x(t),
\end{gathered}
$$

where $\alpha_{i}(z(t))$ 's are the so-called normalized activation functions in relation to the $i$ th submodel such that

$$
\alpha_{i}(z(t))=\frac{\prod_{l=1}^{r} M_{l}^{i}\left(z_{l}(t)\right)}{\sum_{i=1}^{L} \prod_{l=1}^{r} M_{l}^{i}\left(z_{l}(t)\right)}, \quad 0 \leq \alpha_{i}(z(t)) \leq 1 .
$$

Now, we are in the position to develop the global positive stability results for the unforced biochemical network (7); that is, $u=0$.

Theorem 9. If there exists a diagonal matrix $0<P \in \mathbb{R}^{n \times n}$ such that the following LMI conditions

$$
\begin{gathered}
A_{i}^{\mathrm{T}} P+P A_{i}<0, \\
a_{k j}^{i} p_{j j} \geq 0, \quad k, j=1,2, \ldots, n, k \neq j,
\end{gathered}
$$

hold for $i=1,2, \ldots, L$, then the unforced biochemical network (7) is positive and asymptotically stable.

Proof. Construct the following quadratic Lyapunov function candidate for the unforced biochemical network (7)

$$
V(x(t))=x^{\mathrm{T}}(t) P x(t),
$$

where the diagonal matrix $P>0$ is to be determined.
Taking the derivative along the trajectory of (7), one can readily get

$$
\begin{aligned}
\frac{d V(x(t))}{d t}= & \dot{x}^{\mathrm{T}}(t) P x(t)+x^{\mathrm{T}}(t) P \dot{x}(t) \\
= & {\left[\sum_{i=1}^{L} \alpha_{i}(z(t)) A_{i} x(t)\right]^{\mathrm{T}} P x(t) } \\
& +x^{\mathrm{T}}(t) P\left[\sum_{i=1}^{L} \alpha_{i}(z(t)) A_{i} x(t)\right] \\
= & \sum_{i=1}^{L} \alpha_{i}(z(t)) x^{\mathrm{T}}(t)\left[A_{i}^{\mathrm{T}} P+P A_{i}\right] x(t)
\end{aligned}
$$

Then, it follows immediately from condition (9) that

$$
\frac{d V(x(t))}{d t}<0
$$

for all nonzero $x(t)$, and $d V(x(t)) / d t=0$ if and only if $x(t)=0$. Hence, the unforced biochemical network (7) is asymptotically stable.

Furthermore, since $P=\operatorname{diag}\left(p_{11}, \ldots, p_{n n}\right)$ is a positive definite diagonal matrix, that is, $p_{i i}>0, i=1,2, \ldots, n$, it can be deduced from the LMI condition (10) that matrix $A_{i}$ is a Metzler matrix for every local linear model of (7). Moreover, it follows from condition (8) that the membership function satisfies

$$
\sum_{i=1}^{L} \alpha_{i}(z(t))=1, \quad 0 \leq \alpha_{i} \leq 1
$$

which together with the fact that $A_{i}$ is Metzler can guarantee the unforced biochemical network (7) to be positive. Hence, together with condition (13), it can be concluded that the equilibrium point of the unforced biochemical network (7) is positive and asymptotically stable. The proof is thus completed.

Remark 10. It is noted that the obtained sufficient condition for positive stability is in the form of linear matrix inequalities (LMIs), which could be efficiently solved by using the Matlab LMI control toolbox. Compared with the results developed in [20-25], the regulation function is no longer needed to satisfy a sector condition; thus, the regulation function considered here is more general.

\section{Bio-Circuits Design for Nonlinear Biochemical Networks}

If the equilibrium point of the unforced nonlinear biochemical network (7) is not stable, bio-circuits design would become necessary for these complex biological systems to work properly, which would be useful for drug design and gene therapy. In this paper, the following smooth controller is employed to stabilize the biochemical network (7): 


$$
\begin{gathered}
R^{i}: \operatorname{IF} z_{1}(t) \text { is } M_{1}^{i} \text { and } \cdots, z_{r}(t) \text { is } M_{r}^{i} \text {, THEN } \\
u(t)=K_{i} x(t), \quad i \in\{1,2, \ldots, L\}
\end{gathered}
$$

which can be rewritten as

$$
u(t)=\sum_{i=1}^{L} \alpha_{i}(z(t)) K_{i} x(t)
$$

where $K_{i}, i=1,2, \ldots, L$ are the feedback gains of the biocircuits to be determined.

The controlled biochemical network (7) can be described as follows:

$$
\begin{gathered}
\dot{x}(t)=\sum_{j=1}^{L} \sum_{i=1}^{L} \alpha_{j} \alpha_{i}\left(A_{i}+B_{i} K_{j}\right) x(t), \\
y(t)=\sum_{i=1}^{L} \alpha_{i}(z(t)) C_{i} x(t) .
\end{gathered}
$$

Then, the following bio-circuits design results would guarantee the positivity and asymptotic stability of the nonlinear biochemical network (17).

Theorem 11. There exists a smooth control scheme $u(t)=$ $\sum_{i=1}^{L} \alpha_{i}(z(t)) K_{i} x(t)$ such that the biochemical network (17) is positive and asymptotically stable, if there exist a diagonal matrix $0<P \in \mathbb{R}^{n \times n}$ and matrices $Q_{i}, i=1,2, \ldots, L$ such that the following LMI conditions

$$
\begin{gathered}
P A_{i}^{\mathrm{T}}+Q_{j} B_{i}^{\mathrm{T}}+A_{i} P+B_{i} Q_{j}<0, \\
a_{k s}^{i} p_{s s}+\sum_{t=1}^{m} b_{k t}^{i} q_{t s}^{j} \geq 0, \quad k, s=1,2, \ldots, n, k \neq s,
\end{gathered}
$$

hold for $i, j=1,2, \ldots, L$.

Moreover, the feedback gains can be computed as

$$
K_{j}=Q_{j} P^{-1}, \quad j=1,2, \ldots, L .
$$

Proof. Construct the following quadratic Lyapunov function for the biochemical network (17)

$$
V(x(t))=x^{\mathrm{T}}(t) P^{-1} x(t),
$$

where the diagonal matrix $P>0$ is to be determined.
Taking the derivative along the trajectory of system (17), one has

$$
\begin{aligned}
& \frac{d V(x(t))}{d t}= \dot{x}^{\mathrm{T}}(t) P^{-1} x(t)+x^{\mathrm{T}}(t) P^{-1} \dot{x}(t) \\
&= {\left[\sum_{j=1}^{L} \sum_{i=1}^{L} \alpha_{j} \alpha_{i}\left(A_{i}+B_{i} K_{j}\right) x(t)\right]^{\mathrm{T}} P^{-1} x(t) } \\
&+x^{\mathrm{T}}(t) P^{-1}\left[\sum_{j=1}^{L} \sum_{i=1}^{L} \alpha_{j} \alpha_{i}\left(A_{i}+B_{i} K_{j}\right) x(t)\right] \\
&= \sum_{j=1}^{L} \sum_{i=1}^{L} \alpha_{j} \alpha_{i} x^{\mathrm{T}}(t) \\
& \times\left[\left(A_{i}+B_{i} K_{j}\right)^{\mathrm{T}} P^{-1}+P^{-1}\left(A_{i}+B_{i} K_{j}\right)\right] x(t), \\
& \quad i, j=1,2, \ldots, L .
\end{aligned}
$$

It is noted that

$$
\left(A_{i}+B_{i} K_{j}\right)^{\mathrm{T}} P^{-1}+P^{-1}\left(A_{i}+B_{i} K_{j}\right)<0, \quad i, j=1,2, \ldots, L,
$$

which is equivalent to

$$
P\left(A_{i}+B_{i} K_{j}\right)^{\mathrm{T}}+\left(A_{i}+B_{i} K_{j}\right) P<0, \quad i, j=1,2, \ldots, L .
$$

Therefore, it follows immediately from conditions (18) and (20) that

$$
\frac{d V(x(t))}{d t}<0
$$

for all the nonzero $x(t)$, and $d V(x(t)) / d t=0$ if and only if $x(t)=0$. Hence, the controlled biochemical network (17) is asymptotically stable.

On the other hand, since $P$ is a positive definite diagonal matrix, that is, $p_{i i}>0, i=1,2, \ldots, n$, it follows from conditions (19) and (20) that the off-diagonal elements of matrix $\left(A_{i}+B_{i} K_{j}\right)$ are nonnegative; that is,

$$
\begin{aligned}
a_{k s}^{i}+\frac{\sum_{t=1}^{m} b_{k t}^{i} q_{t s}^{j}}{p_{s s}} & =a_{k s}^{i}+\sum_{t=1}^{m} b_{k t}^{i} k_{t s}^{j} \geq 0, \\
k, s & =1,2, \ldots, n, \quad k \neq s, \quad i, j=1,2, \ldots, L .
\end{aligned}
$$

This implies that for any $i, j=1,2, \ldots, L, A_{i}+B_{i} K_{j}$ is a Metzler matrix for every linear local model. Similar to the proof of Theorem 9, from condition (14), it can be concluded that the controlled biochemical network (17) is positive. Therefore, together with condition (25), one can conclude that the controlled biochemical network (17) is positive and asymptotically stable. The proof is, thus, completed. 
It is worth pointing out that in many practical applications, drugs or chemical complexes delivered to the human body are often taken as control inputs to biochemical systems, and in this case, control signals have to be nonnegative. Therefore, it is necessary to consider the positive constraints of control inputs when designing bio-circuits. We have the following results.

Theorem 12. There exists a positive smooth control scheme $u(t)=\sum_{i=1}^{L} \alpha_{i}(z(t)) K_{i} x(t)$, that is, $0 \preceq u(t)$, such that the biochemical network (17) is positive and asymptotically stable, if there exist a diagonal matrix $0<P \in \mathbb{R}^{n \times n}$ and matrices $Q_{i}, i=1,2, \ldots, L$ such that the following LMI conditions

$$
\begin{gathered}
P A_{i}^{\mathrm{T}}+Q_{j} B_{i}^{\mathrm{T}}+A_{i} P+B_{i} Q_{j}<0, \\
a_{k s}^{i} p_{s s}+\sum_{t=1}^{m} b_{k t}^{i} q_{t s}^{j} \geq 0, \quad k, s=1,2, \ldots, n, k \neq s, \\
q_{t s}^{j} \geq 0, \quad t=1,2, \ldots, m, s=1,2, \ldots, n, \\
\text { hold for } i, j=1,2, \ldots, L .
\end{gathered}
$$

Moreover, the feedback gains can be computed as

$$
K_{j}=Q_{j} P^{-1}, \quad j=1,2, \ldots, L .
$$

Remark 13. It is noted that the positive feedback control law is only available in some special cases. When $A_{i}$ is a Metzler matrix and $B_{i} \geq 0$, it is impossible to design a positive feedback bio-circuit.

Remark 14. In many practical applications, the concentrations of some materials should be rigorously kept below or above a certain level, otherwise they may have side effects on blood or other chemical complexes. In other words the states of biochemical networks should be subject to some kinds of constraints. How to deal with such scenarios is one of our future research interests.

\section{Design Example In Silico for the Proposed Method}

Consider a two-compartment model [31], which describes the kinetics of a drug in the human body. The drug is injected into the blood where it exchanges linearly with the tissues; the drug is irreversibly removed with a nonlinear saturative characteristic from the blood and with a linear one from the tissue. The model can be expressed by the following nonlinear differential equations:

$$
\begin{gathered}
\dot{x}_{1}=-\left(a_{11}+\frac{V_{M}}{V_{m}+x_{1}}\right) x_{1}+a_{12} x_{2}+b_{1} u, \\
\dot{x}_{2}=a_{21} x_{1}-\left(a_{02}+a_{22}\right) x_{2}, \\
y=c_{1} x_{1},
\end{gathered}
$$

where $x_{1}, x_{2}$ are the drug masses in blood and tissues, respectively; $u$ is the drug input $y$ is the measured drug output in the blood; $a_{11}, a_{12}, a_{21}, a_{22}$, and $a_{02}$ are the constant rate parameters; $V_{M}$ and $V_{m}$ are the Michaelis-Menten parameters; $b_{1}$ and $c_{1}$ are the input and output parameters, respectively.

Let the premise variable $z_{1}(t)=x_{1}(t)$, then the membership functions can be chosen as

$$
M_{1}^{1}\left(z_{1}(t)\right)=\frac{V_{m}}{V_{m}+x_{1}(t)}, \quad M_{1}^{2}\left(z_{1}(t)\right)=\frac{x_{1}(t)}{V_{m}+x_{1}(t)} .
$$

By using $M_{1}^{1}$ and $M_{1}^{2}$, the biochemical network (29) can be expressed by the following T-S fuzzy model

$$
\begin{aligned}
& \text { Plant Rule 1: IF } z_{1}(t) \text { is } M_{1}^{1}, \text { THEN } \\
& \begin{array}{c}
\dot{x}(t)=A_{1} x(t)+B_{1} u(t), \\
y(t)=C_{1} x(t),
\end{array}
\end{aligned}
$$

Plant Rule 2: IF $z_{1}(t)$ is $M_{1}^{2}$, THEN

$$
\begin{gathered}
\dot{x}(t)=A_{2} x(t)+B_{2} u(t), \\
y(t)=C_{2} x(t),
\end{gathered}
$$

where $x(t)=\left[x_{1}(t), x_{2}(t)\right]^{\mathrm{T}}$ and

$$
\begin{gathered}
A_{1}=\left[\begin{array}{cc}
-\left(a_{11}+V_{M} / V_{m}\right) & a_{12} \\
a_{21} & -\left(a_{02}+a_{22}\right)
\end{array}\right], \\
B_{1}=\left[\begin{array}{c}
b_{1} \\
0
\end{array}\right], \quad C_{1}=\left[\begin{array}{ll}
c_{1} & 0
\end{array}\right] ; \\
A_{2}=\left[\begin{array}{cc}
-a_{11} & a_{12} \\
a_{21} & -\left(a_{02}+a_{22}\right)
\end{array}\right], \\
B_{2}=\left[\begin{array}{c}
b_{1} \\
0
\end{array}\right], \quad C_{2}=\left[\begin{array}{ll}
c_{1} & 0
\end{array}\right] .
\end{gathered}
$$

Let $a_{11}=0.1, a_{21}=2, a_{12}=0.5, a_{22}=0.1, a_{02}=$ $0.6, V_{M}=1, V_{m}=0.5$, and $b_{1}=-1$; Figure 1 shows that the state trajectories of biochemical network (29) are divergent under $u=0$ with the initial condition $x(0)=[0.1,4]^{\mathrm{T}}$. For convenience, we use the same initial conditions in the following simulations.

5.1. Bio-Circuits Design without Constraints. In this case study, we will consider the bio-circuits design for the biochemical network (31) without constraints. Solving the LMI conditions (18)-(19) leads to the feasible solutions as follows:

$$
\begin{gathered}
P=\left[\begin{array}{cc}
8.5971 & 0 \\
0 & 39.6566
\end{array}\right], \\
Q_{1}=Q_{2}=\left[\begin{array}{ll}
34.1204 & -10.3198
\end{array}\right] .
\end{gathered}
$$

Then the feedback gains can be calculated as $K_{1}=K_{2}=$ $[3.9688,-0.2602]$, which could guarantee the controlled biochemical network (31) to be positive and asymptotically stable. The dynamic response of the controlled biochemical network can be seen in Figure 2, and the evolution of the control input can be seen in Figure 3. It can be observed that the control input is negative in some stage. 


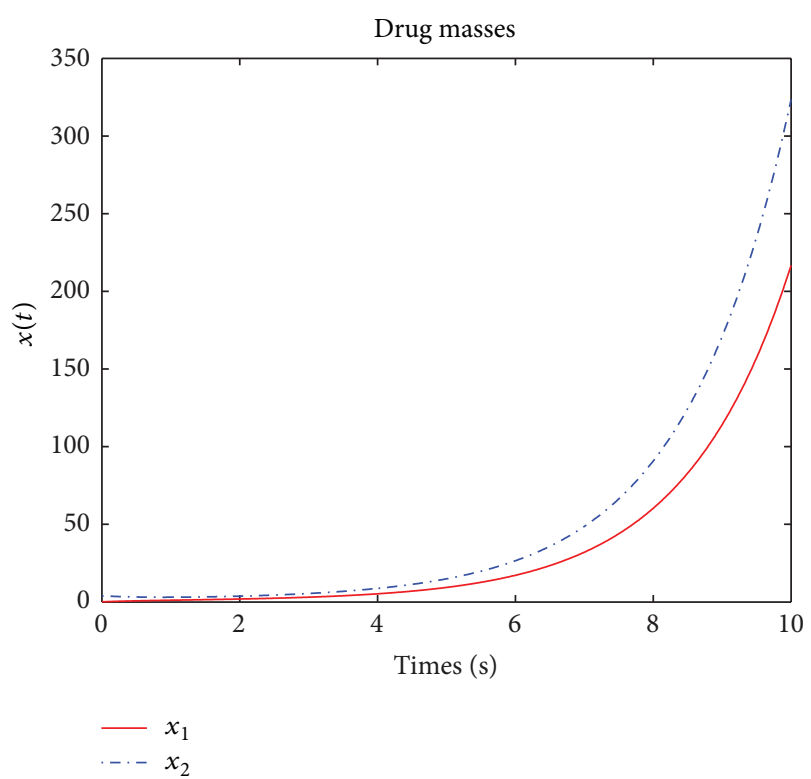

FIgURE 1: Time response of the biochemical network under $u=0$.

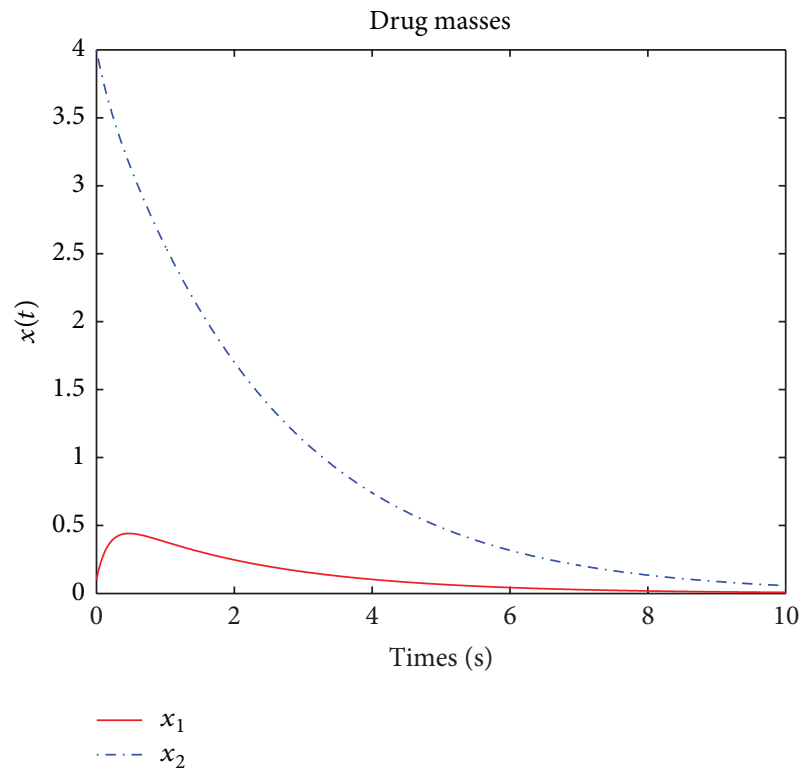

FIgURE 2: Time response of the controlled biochemical network.

5.2. Bio-Circuits Design with Positive Control Constraints. In most situations, the control input $u(t)$ is the drug or the mixture of some biochemical complexes, which should be constrained to be positive. From Theorem 12, the elements of nonsymmetric matrixes $Q_{1}, Q_{2}$ should satisfy conditions (27). By using the constrained LMI algorithm, the following feasible solutions are obtained:

$$
\begin{gathered}
P=\left[\begin{array}{cc}
1.1517 & 0 \\
0 & 9.6618
\end{array}\right], \\
Q_{1}=Q_{2}=\left[\begin{array}{ll}
5.3937 & 3.7805
\end{array}\right] .
\end{gathered}
$$

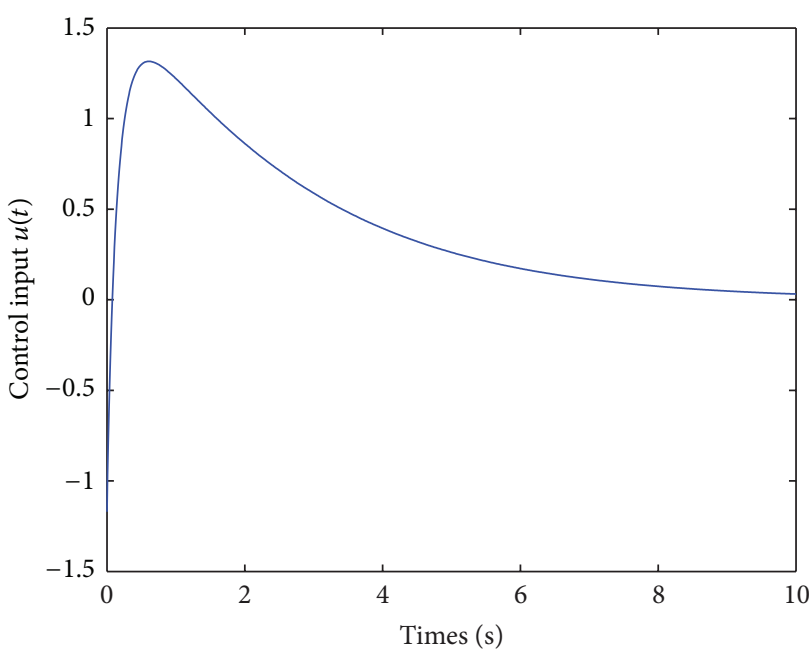

FIGURE 3: Evolution of the control input without constraints.

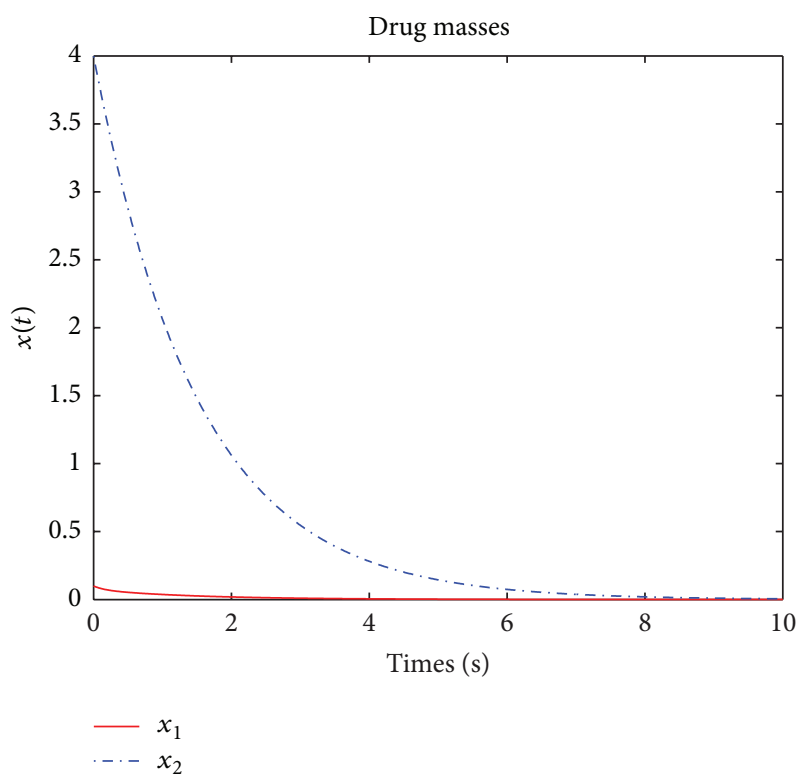

FIGURE 4: Time response of the biochemical network under positive control.

Then, the feedback gains can be calculated as $K_{1}=K_{2}=$ $[4.6831,0.3913]$. The state response of the biochemical network under this constrained control can be seen in Figure 4, and the evolution of the positive control input $u(t)$ can be seen in Figure 5, where the control signal remains to be positive all the time.

Remark 15. By using the fuzzy interpolation approach, biocircuits can be easily implemented for the nonlinear biochemical network (29), which could guarantee it to be positive and asymptotically stable. Although the stability conditions for this biochemical network can also be derived by the method proposed in [29], the positivity of the states cannot be guaranteed. This would greatly reduce the significance of those results in real application such as drug delivery. 


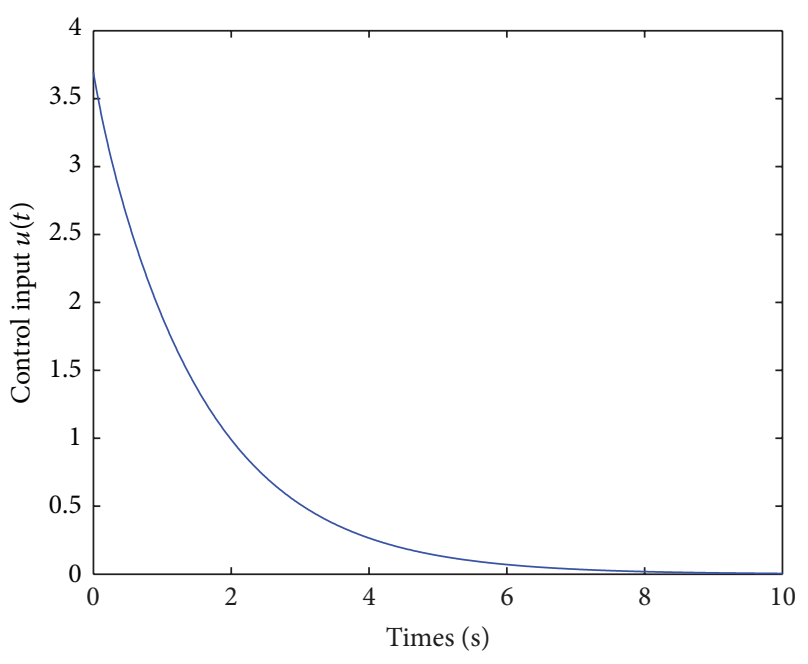

Figure 5: Evolution of the control input with constraints.

\section{Discussion}

In this paper, the fuzzy interpolation approach has been employed to approximate nonlinear biochemical networks for positive stability analysis and bio-circuits design. A few results on sufficient conditions for positivity and asymptotic stability of the network have been obtained in terms of a number of linear matrix inequalities. In addition, the positive constraint on control input is also considered for bio-circuits design. Finally, a real biochemical network model was provided to illustrate the effectiveness and validity of the obtained results.

Due to the transcription, translation, diffusion, and translocation processes of genes, time delays are inevitable in describing the dynamics of biochemical networks [7]. In addition, biochemical networks are often subject to intrinsic and extrinsic perturbations such as gene expression noises, mutation and disturbance from uncertain environment, and the fractal and chaotic features of systems [32]. Therefore, how to design constrained robust bio-circuits for such biochemical networks will be an interesting and challenging task.

\section{Acknowledgment}

This work was supported in part by the National Natural Science Foundation of China under Grants 61104045, 51107032, and 51277052, and in part by the Fundamental Research Funds for the Central Universities of China under Grant 2012B03514.

\section{References}

[1] E. R. Carson, C. Cobelli, and L. Finkelstein, The Mathematical Modeling of Metabolic and Endocrine Systems, John Wiley \& Sons, New York, NY, USA, 1983.

[2] H. de Jong, "Modelling and simulation of genetic regulatory systems: a literature review," Journal of Computational Biology, vol. 9, no. 1, pp. 67-103, 2002.
[3] J. Hasty, M. Millen, and J. J. Collins, "Engineered gene circuits," Nature, vol. 420, no. 6912, pp. 224-230, 2002.

[4] E. D. Sontag, "Molecular systems biology and control," European Journal of Control, vol. 11, no. 4-5, pp. 396-435, 2005.

[5] E. Klipp, R. Herwig, A. Kowald, C. Wierling, and H. Lehrach, Systems Biology in Practice: Concepts, Implementation and Application, John Wiley \& Sons, New York, NY, USA, 2005.

[6] P. D'haeseleer, S. Liang, and R. Somogyi, “Tutorial on gene expression data analysis and modeling," in Proceedings of the Pacific Symposium on Biocomputing, January 1999.

[7] P. Smolen, D. A. Baxter, and J. H. Byrne, "Mathematical modeling of gene networks," Neuron, vol. 26, no. 3, pp. 567-580, 2000.

[8] F. X. Wu, W. J. Zhang, and A. J. Kusalik, "Modeling gene expression from microarray expression data with state-space equations," Pacific Symposium on Biocomputing, vol. 9, pp. 581592, 2004.

[9] Y. Sun, G. Feng, and J. Cao, "A new approach to dynamic fuzzy modeling of genetic regulatory networks," IEEE Transactions on Nanobioscience, vol. 9, no. 4, pp. 263-272, 2010.

[10] H. Xiong and Y. Choe, "Structural systems identification of genetic regulatory networks," Bioinformatics, vol. 24, no. 4, pp. 553-560, 2008.

[11] D. Angeli, J. E. Ferrell, and E. D. Sontag, "Detection of multistability, bifurcations, and hysteresis in a large class of biological positive-feedback systems," Proceedings of the National Academy of Sciences of the United States of America, vol. 101, no. 7, pp. 1822-1827, 2004.

[12] J. Keener and J. Sneyd, Mathematical Physiology, Springer, New York, NY, USA, 1998.

[13] L. Hood, J. R. Heath, M. E. Phelps, and B. Lin, "Systems biology and new technologies enable predictive and preventative medicine," Science, vol. 306, no. 5696, pp. 640-643, 2004.

[14] L. Farina and S. Rinaldi, Positive Linear Systems: Theorey and Applications, John Wiley \& Sons, New York, NY, USA, 2000.

[15] H. Gao, J. Lam, C. Wang, and S. Xu, "Control for stability and positivity: equivalent conditions and computation," IEEE Transactions on Circuits and Systems II, vol. 52, no. 9, pp. 540544, 2005.

[16] M. A. Rami and F. Tadeo, "Controller synthesis for positive linear systems with bounded controls," IEEE Transactions on Circuits and Systems II, vol. 54, no. 2, pp. 151-155, 2007.

[17] X. Liu, L. Wang, W. Yu, and S. Zhong, "Constrained control of positive discrete-time systems with delays," IEEE Transactions on Circuits and Systems II, vol. 55, no. 2, pp. 193-197, 2008.

[18] J. A. Jacquez and C. P. Simon, "Qualitative theory of compartmental systems," SIAM Review, vol. 35, no. 1, pp. 43-79, 1993.

[19] W. M. Haddad and V. Chellaboina, "Stability and dissipativity theory for nonnegative dynamical systems: a unified analysis framework for biological and physiological systems," Nonlinear Analysis: Real World Applications, vol. 6, no. 1, pp. 35-65, 2005.

[20] L. Chen and K. Aihara, "Stability of genetic regulatory networks with time delay," IEEE Transactions on Circuits and Systems I, vol. 49, no. 5, pp. 602-608, 2002.

[21] C. Li, L. Chen, and K. Aihara, "Stability of genetic networks with SUM regulatory logic: lur'e system and LMI approach," IEEE Transactions on Circuits and Systems I, vol. 53, no. 11, pp. 24512458, 2006.

[22] C. Li, L. Chen, and K. Aihara, "Stochastic stability of genetic networks with disturbance attenuation," IEEE Transactions on Circuits and Systems II, vol. 54, no. 10, pp. 892-896, 2007. 
[23] F. Ren and J. Cao, "Asymptotic and robust stability of genetic regulatory networks with time-varying delays," Neurocomputing, vol. 71, no. 4-6, pp. 834-842, 2008.

[24] Z. Wang, H. Gao, J. Cao, and X. Liu, "On delayed genetic regulatory networks with polytopic uncertainties: robust stability analysis," IEEE Transactions on NanoBioscience, vol. 7, no. 2, pp. 154-163, 2008.

[25] Y. Sun, G. Feng, and J. Cao, "Robust stochastic stability analysis of genetic regulatory networks with disturbance attenuation," Neurocomputing, vol. 79, pp. 39-49, 2012.

[26] T. Takagi and M. Sugeno, "Fuzzy identification of systems and its applications to modeling and control," IEEE Transactions on Systems, Man, and Cybernetics, vol. 15, no. 1, pp. 116-132, 1985.

[27] B. S. Chen, Y. T. Chang, and Y. C. Wang, "Robust Hostabilization design in gene networks under stochastic molecular noises: fuzzy-interpolation approach," IEEE Transactions on Systems, Man, and Cybernetics B, vol. 38, no. 1, pp. 25-42, 2008.

[28] J. Hasty, J. Pradines, M. Dolnik, and J. J. Collins, "Noise-based switches and amplifiers forgene expression," Proceedings of the National Academy of Sciences of the United States of America, vol. 97, no. 5, pp. 2075-2080, 2000.

[29] B. S. Chen, W. S. Wu, Y. C. Wang, and W. H. Li, "On the robust circuit design schemes of biochemical networks: steadystate approach," IEEE Transactions on Biomedical Circuits and Systems, vol. 1, no. 2, pp. 91-104, 2007.

[30] B. S. Chen and P. W. Chen, "Robust engineered circuit design principles for stochastic biochemical networks with parameter uncertainties and disturbances," IEEE Transactions on Biomedical Circuits and Systems, vol. 2, no. 2, pp. 114-132, 2008.

[31] M. J. Chappell and K. R. Godfrey, "Structural identifiability of the parameters of a nonlinear batch reactor model," Mathematical Biosciences, vol. 108, no. 2, pp. 241-251, 1992.

[32] F. Wen, Z. Li, C. Xie, and D. Shaw, "Study on the fractal and chaotic features of the Shanghai composite index," Fractals, vol. 20, no. 2, pp. 133-140, 2012. 


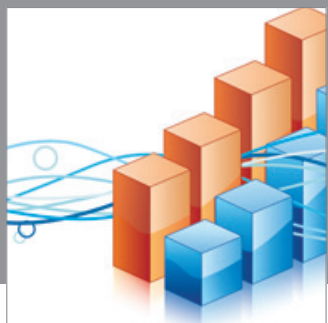

Advances in

Operations Research

mansans

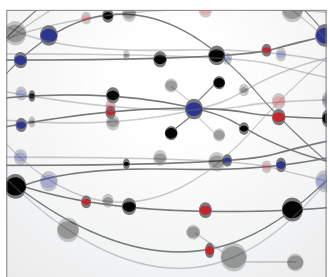

The Scientific World Journal
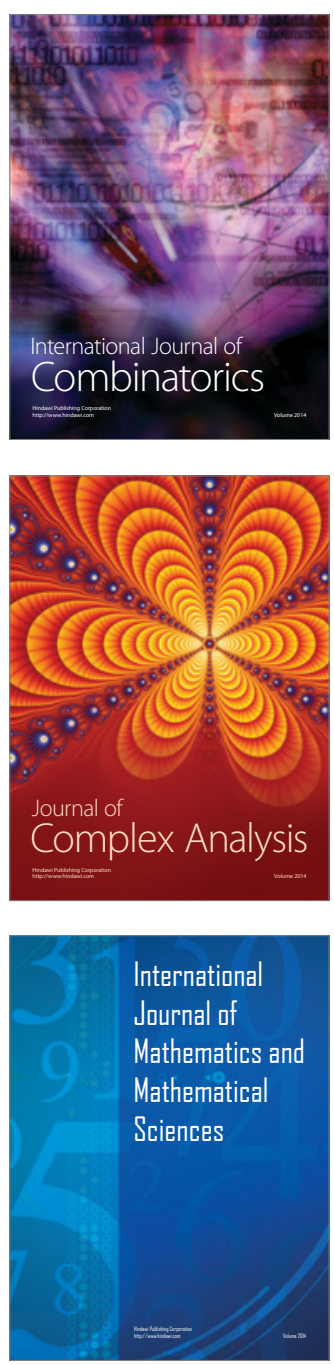
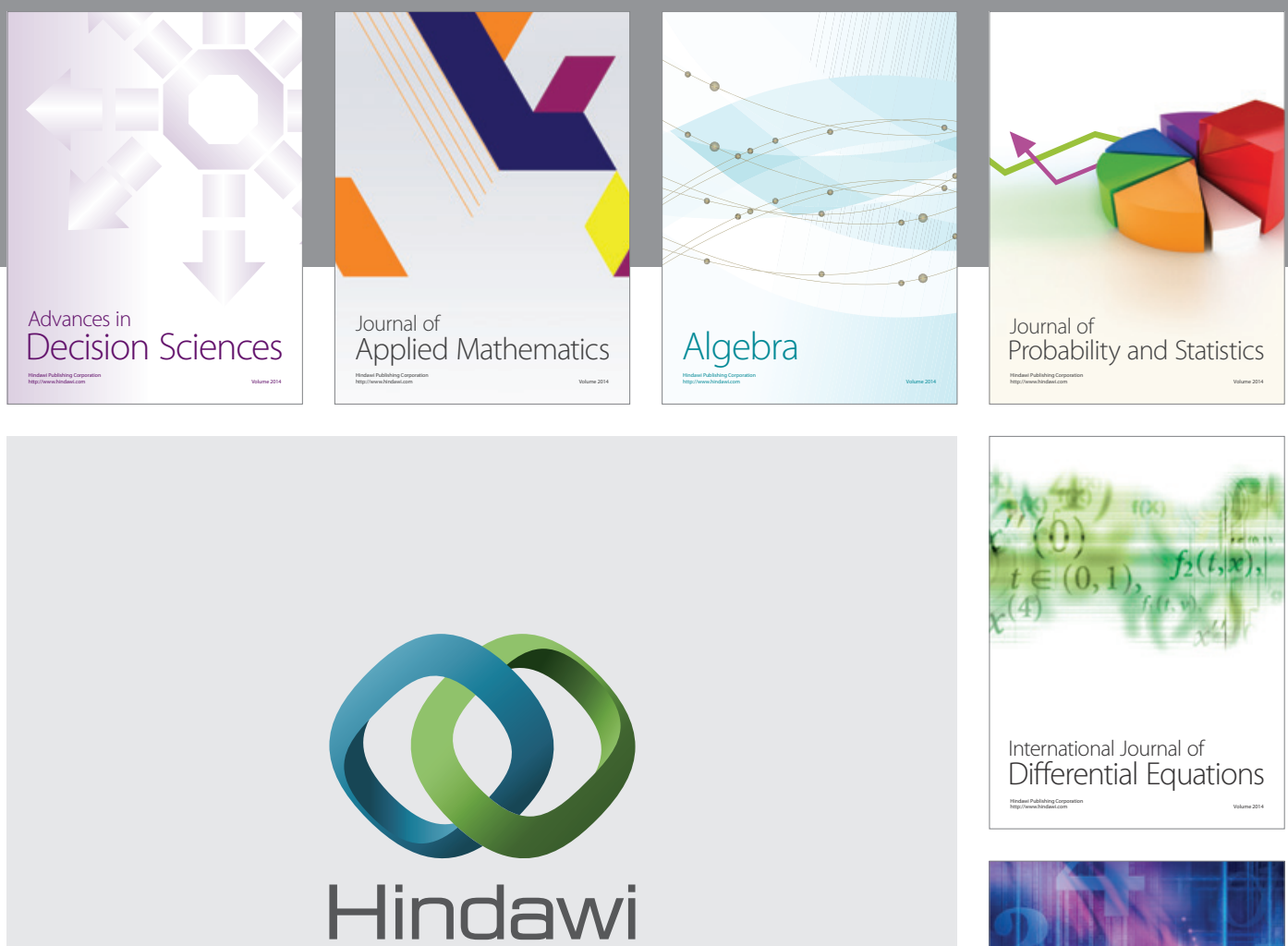

Submit your manuscripts at http://www.hindawi.com
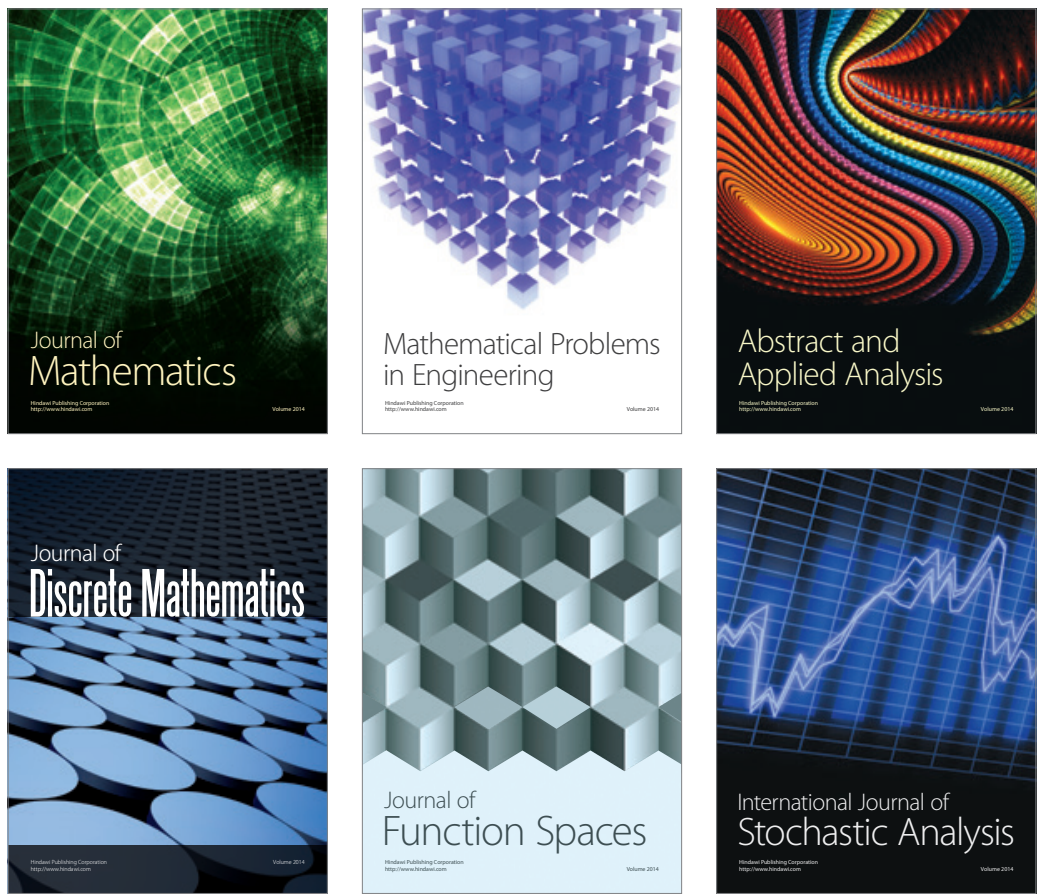

Journal of

Function Spaces

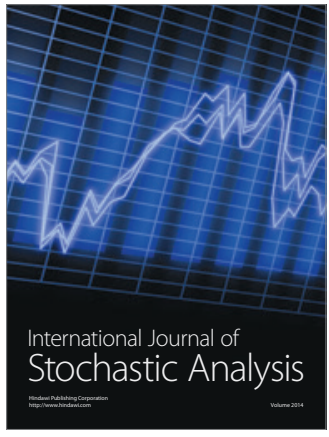

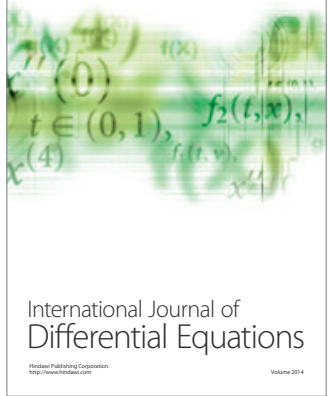
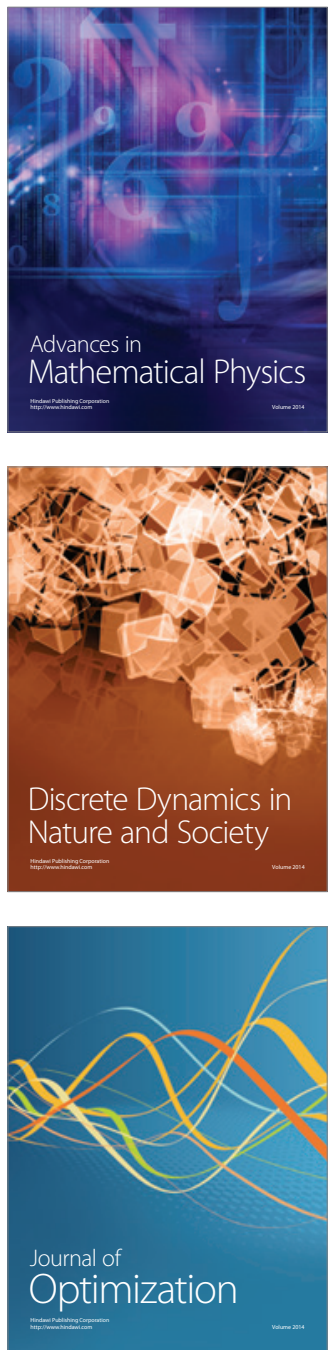